\title{
Keys to Promoting Governmental Digital Transformation
}

\section{Hsiu-Ling Li*}

Market Intelligence \& Consulting Institute (MIC), Institute for Information Industry (III), Taiwan

(Received 9 November 2018; Published on line 1 December 2018)

*Corresponding author email: linahlli@micmail.iii.org.tw

DOI: 10.5875 /ausmt.v8i4.2066

Digital technology is continually advancing and speedily permeating into our daily lives and interactions, changing the way we work, learn, and socialize. New commercial models are also changing the management models of enterprises. Aside from the digital transformation issues that industries are facing, governance from the government must also keep up with the times and have the citizens' needs close to heart. A more innovative mindset is needed to design and dispatch services, providing what citizens need and perhaps more than what they expect. The UK and the US noted the trend of digital development quite early on and dictated digital transformation as a priority strategy nationwide. This report aims to sort out the keys to successful digital transformation and possible issues arising from the UK and the US's transformation into digital governments ${ }^{1}$. The understanding of the strategies and methods used can serve as a reference for Taiwan to follow, as to accelerate successful digital transformation of the local government.

From the development history of governmental digital transformation by the UK and the US, five common characteristics can be found:

\section{Explicit development strategy roadmaps}

Digital government strategy was proposed in the UK in 2012; 11 principles and 14 specific actions were drawn

\footnotetext{
${ }^{1}$ According to the definition of a digital government in OECD's Recommendation of the Council on Digital Government Strategies report in 2014, digital government is part of the modernization strategy of governments. This refers to governments utilizing digital technology to create public value, and relying on government organizations, non-government
}

up to make the government "digital by default". All government departments were also required to draw up their own departmental digital strategies in reference to the main strategy. This went to ensure that the digital services provided were easy-to-use and convenient for all citizens. To help government departments improve digital services, the central government also established digital assets, including standards and design principles for access by all departments. Moreover, not all citizens make use of the Internet. In 2012, 18\% of Britons had still never or rarely used the Internet ${ }^{2}$. To deal with these citizens, the UK government provided assisted digital support services, as in face-to-face and telephone services. In 2017 a new digital strategy was announced, pointing out the key focuses of the next phase of digital transformation, including continuing to develop world-class digital services; cultivating the right talent, skills and culture; creating better tools, procedures and governance; making good use of data; and speeding up transformation of a new sharing platform.

In 2012 the US also announced a 21st century digital government strategy, unveiling a year-long plan to build the basic infrastructure necessary for the digital government in the future. This infrastructure had three layers to it, an information layer, platform layer and presentation layer. Separating data creation from data presentation enabled new ways of presenting the content and data created. The strategy was based on 4 principles:

organizations, enterprises, and the digital government ecosystem formed by citizen communities and individuals. Data, services and content are created through interactions with the government, increasing public satisfaction towards using public services.

2 UK Internet Census, Aug 2012, Cabinet Office. 
Information-centric, Shared platforms, Customer-centric, and Security \& privacy. Through the key paths planned, the government continued towards the road of unleashing the full potential of digital transformation and becoming a highly effective government dedicated to serving the American people.

\section{Establishing a responsible digital transformation institution}

To proceed with transformation tasks, the UK government created a Government Digital Services (GDS) team under the Cabinet Office in 2011. Besides drafting a digital strategy, the UK government also specifically assisted cross-departmental digital transformation by establishing platforms, standards and digital services with all departments. The establishment of GDS was tailored to users' requirements, with emphasis on agility and openness, and was dedicated to establishing a set of principles as guidance for GDS itself and other departments' transformation tasks. From 2012 to 2015, the way GDS assisted all departments' digital transformation was by establishing 25 demo items to accelerate all departments' learning and replication. In 2014, the Digital Academy was established to explain to the digital workers of all government departments how to build services for users with new methods. A series of lectures was then designed to help civil servants upgrade their digital skills to adapt to the needs of transformation tasks.

In 2012, the US announced the 21st century digital government strategy, and the Presidential Innovation Fellows project (PIF) was initiated. Entrepreneurs, CEOs, technicians, designers and other innovators were brought into the government to improve a program that served over 150 million American citizens. In 2014, the United States Digital Service (USDS) was established as a missionbased project organization (cross-departmental team) with 6 component teams including the PIF. These were sixmonth-term paid positions in which top talents were

Ms. Hsiu-Ling Li's research areas include national innovation system, science
and technology policy, and regional development strategic planning, as well as
innovation strategy of the firm. Ms. Li has participated in the projects of "2025
Taiwan Industry and Advanced Technology Research Project", "National
Development Prospective Planning", "The Swiss economy, the industrial
growth model and competitiveness", and "Research of Metropolitan
Innovation Industry Parks". Ms. Li holds the Ph.D. degree in business from
National Taiwan University.
E mail: linahlli@micmail.iii.org.tw

${ }^{3}$ The White House proposed the Playbook with 13 key ways of providing digital government service from industries and government sources, as to assist the government in establishing effective digital services, including: Understand what people need; Address the whole experience, from start to finish; Make it simple and intuitive; Build the service using agile and iterative externally hired to act as advisors for the government, and made many contributions on important issues to create digital services. When a project was proposed, it could be planned from cross-departmental and cross-agency perspectives. IT managers involved in the project were factually influential and could start the changes at their respective institutions.

\section{Establishment of shared platform and standard}

To make the speed of transformation amongst the departments even faster, the UK's GDS ideally made the government a platform to develop common technology, platforms and components for all departments to share and utilize, easily building "user-centered" digital services. Currently, five components are in place: GOV.UK Verify, GOV.UK Notify, GOV.UK Pay, GOV.UK Platform as a Service, and Registers. GOV.UK, a single government portal website built by the GDS, played an important role for digital transformation of the UK government. A "digital by default" service standard was established; all transaction services accessible to the public must adhere to 18 digital service standards. Only departmental services adhering to these service guidelines appear on the GOV.UK site. GOV.UK is estimated to have saved the UK 60 million pounds between 2013-2014.

Taxpayer money in the US goes to waste because of unplanned and inefficient procurement and development methods, and also hinders the use of new technology and methods. Under the shared platform strategy principle, when a new project is developed, all institutions must first consider shared solutions and current infrastructure, and not purchase a new infrastructure and system for each new project. All departments must commonly own the rights to the intra-organization and interdepartmental common service areas, and not build multiple websites based on the same theme. A Digital Services Playbook ${ }^{3}$ was published on the shared platform and standard. A uniform identity management platform was developed and a webpage design standard published. A digital analysis dashboard was also developed.

\section{Focus on key service upgrades}

To make digital services simpler, clearer and faster, the UK government created twenty-five demo services to

practices; Structure budgets and contracts to support delivery; Assign one leader and hold that person accountable; Bring in experienced teams; Choose a modern technology stack; Deploy in a flexible hosting environment; Automate testing and deployments; Manage security and privacy through reusable processes; Use data to drive decisions; and Default to open. 
start off. The chosen demo services are the ones that have served over 10 million persons annually, and are redesigned by capable experienced service managers with the relevant skills. Demo services are based on users' needs. Each demo service is rated on a scale of 1 to 9 , indicating the digital skill level required for the user to complete the services, as to discern the complexity of the services. Rating categories include the average time required to complete the services, necessary information, and the difficulty of using services on a portable device.

The USDS uses the best product design and engineering to change the way the U.S. government serves the American people. Gathering a team that is most capable of solving problems in the US to provide key services, e.g., medicare, student loans, veterans' welfare and twelve others. The goal of upgrading the services is to make them as easy as buying a book online.

\section{Innovative government procurement services}

Public procurement reforms are an important part of the digital government vision in the UK. This stems from the government's past experience of developing IT services utilizing a linear method, which was timeconsuming and inflexible. The UK proposed adopting a more agile approach in developing an IT tech procuring manual, thus dividing development into three phases: Alpha, Beta and Live. All three phases have rolling development testing of design, building, testing, and deployment for the product to meet the public's needs more closely, and to avoid walking down the same path of previous major projects. The UK government also cooperated with Crown Commercial Service to collect all information on collaborating suppliers. All government departments can search for personnel and technology necessary for projects in the digital marketplace, which makes for a diversification of digital and technical suppliers. In 2022, the UK plans for a third of product and service procurements to be done directly or through a supply chain in cooperation with small and medium enterprises (SMEs).

Through establishing a professional procurement taskforce, the USDS improved the technical market for the federal government. Digital services help the departments make the best purchasing decisions. Ways of improvement include: 1 . Simplifying the small enterprise authentication process to provide more opportunities for small enterprises to enter the federal government procurement market; 2 . Developing agile procurement tools like Tech FAR Hub to provide a point of interface for digital service procurement personnel, helping the government consistently procure high quality products; 3 . Developing digital IT procurement expert communities through a Digital Service Procurement Personnel Training Challenge Program, and recruiting creative talent for training programs on Challenge.gov, so that federal procurement personnel can catch up with the digital age. Winners of challenges can become digital service procurement sales consultants for the government after undergoing training.

\section{Problems encountered during the transformation process}

When going ahead with the digital transformation, the UK and the US governments actually ran into numerous problems. For example the UK National Audit Office (NAO) points out that although the GDS successfully reshaped the government's approach towards technology and transformation, there are still some risks associated. The overexpansion of GDS has led to responsibilities not being clearly laid out. The report also points out that, in 2015, GDS received a four-year funding of 455 million pounds (2016-2020) but there was no actual plan regarding spending of that funding. Moreover, in 2012, GDS assisted all departments in redesigning 25 important and most frequently used public services, aiming to provide the public with more convenient government services and cutting unnecessary costs. And the 2015 NAO report shows that, with normal data accessibility, only 12 of the 22 available services pulled in a net profit. The GDS has claimed its new services and the GOV.UK portal site can save the government up to 1.7 billion pounds in service costs, but that would only be possible if civil servants could be asked to resign due to citizens' capability of self-service. NAO has expressed doubt over this, and has requested GDS twice to lower their targets and to base them on cold hard facts.

The identification verification platform GOV.UK Verify was rolled out in May 2015. Individual users can safely utilize digital government services with reliable and safe online identity verification, such as declaring taxes or checking information on a driver's license. This aims to prevent fraud and protect users' privacy. Government statistics in February 2018 show that only $30 \%$ of users are able to successfully use GOV.UK Verify, well below the estimated $80 \%$. The NAO points out that the GDS should understand the current state of operations of all departments, because in the past there was no comprehensive analysis on how current services identify customers, how customer information is held within these services, or what possibly affects customers from verification to the completion of a transaction. These analysis results can provide an impetus for departments to start using Verify.

Another example would be the log-on problems at HealthCare.gov from the US. With the signing into law of 
the Patient Protection and Affordable Care Act (PPACA) by President Barack Obama, the US government spent $\$ 678$ million building the federal health exchange website HealthCare.gov. The site was officially launched on October 1, 2013. American citizens were able to register on the federal government or state healthcare exchange market. Catastrophe ensued, and on the first day of registration, the site crashed. Only six people registered successfully. The first day the site went online there were over 2.8 million visitors, and 250,000 users were online simultaneously. The website could not handle the tremendous flow of traffic. Only $1 \%$ of users could complete their inquiries and submit their information in the week after the site went online. Healthcare insurance providers also stated that much of the information they received was not complete at all. The painful lesson from HealthCare.gov facilitated the creation of the USDS to try and drastically improve the tech aspect of the government. The technical team was assembled like an internal startup, becoming part of a government organization. "Agile development" model became the standard model for later government development services and accelerating software development.

\section{Key thoughts regarding governmental digital transformation}

To summarize some key points regarding the developmental experience of the US and the UK governments' digital transformation worthy for Taiwan to follow: Firstly, when drafting a direction for strategy, it must begin from the perspective of the citizens' needs. There should also be clear priorities for selection, as to concentrate on the most important issues and build citizen-friendly digital services. Secondly, high level governmental commitments and resource support are also required. For example, the UK set up a GDS under the Cabinet Office to provide budget support and assist all departments in drafting digital strategies. The role of digital leaders was also set up in the various departments. Senior civil servants who had previously led massive internal or external reform projects within the government were the contact persons who coordinated digital activity and enhanced the participation of each department. Thirdly, digital service development must emphasize the participation of the user during the designing phase. An agile development model with a quick response to changes and continuous development is necessary to gradually develop and verify the needs of users that should be implemented. Fourthly, elite technology talent must be simultaneously recruited into the digital transformation team to create a digital civil service system. For example, the UK invented a fast promotion system for digital talent and digital skill courses to support civil servants' digital skill development.

\section{References}

[1] Cabinet Office (2012), Government digital strategy.

[2] Challenge.gov, https://challenge.gov/a/buzz/challenge.

[3] GOV.UK (2012), Government design principles.

[4] GOV.UK (2014), Case study on action2: redesigning services.

[5] GOV.UK (2017), Government Transformation Strategy: platforms, components and business capabilities.

[6] National Audit Office [NAO ] (2017), Digital transformation in government.

[7] The Institute for Government (2016), Making a success of digital government.

[8] US Digital Service, https://www.usds.gov.

[9] US Digital Service, TechFAR Hub, https://techfarhub.cio.gov/.

[10] US Government Accountability Office [GAO ] (2015), CMS Has Taken Steps to Address Problems, but Needs to Further Implement Systems Development Best Practices.

[11] U.S. Small Business Administration [SBA], https://certify.sba.gov/.

[12] US White House Office (2012), Digital government: Building a 21st century platform to better serve the American people.

[13] US White House Office (2016), Impact report: Transforming government services through technology and innovation.

[14] US White House Office, US Digital Services Playbook.

(cc) BY-NC-ND CThe Authors. This work is licensed under the Creative Commons Attribution-NonCommercial-NoDerivatives 4.0 International License. 